\title{
Proceso de puesta en valor para la reutilización del Fuerte de Santiago (Isla de Chikly, Túnez) María José Viñals, Imma Martínez-Sanchis
}

Universitat Politècnica de València, València, España, mvinals@upv.es

\begin{abstract}
Historic defence buildings have intrinsic value in their own right as the fabric of human achievement in arts, design and construction but they were built for a use that no longer exists today and, therefore, they are closed, even administratively abandoned, and the passage of time has caused their deterioration.

It is known that the best way to conserve historic buildings is to find viable and compatible new uses that all historical buildings to retain their historic character conserve significant fabric and keep them in good repair into the future. This idea lies into the concept of the heritage adaptive reuse and successful experiences regarding the transformation of defence sites to civilian uses exist around the world.

This paper presents the results of the enhancement project and reuse of the Spanish Fort of Santiago, located in the Chikly Island (Tunis), where the preliminary studies (recreational carrying capacity, tour pattern, accessibility, etc.) have been performed in order to be visited.
\end{abstract}

Keywords: Adaptive reuse, fortification, enhancement, recreational carrying capacity.

\section{Introducción}

Los bienes arquitectónicos son el reflejo de la sociedad que los construye. Con el paso del tiempo y los avances culturales y tecnológicos, la sociedad precisa de espacios para nuevas funciones, al tiempo que otras construcciones quedan en desuso por su pérdida de funcionalidad, disminución de rendimiento o por motivos económicos, llegando en muchos casos a la desaparición. Esta dinámica afecta igualmente a los edificios históricos que forman parte del patrimonio cultural de una nación.

Tanto en la literatura científica (Canada's Historic Places, 2010; Chapple, 2012) como en los manifiestos o cartas internacionales referentes a la conservación del patrimonio (UNESCO, 1964; 1972; 1999) se expresa que la forma más efectiva de conservar el patrimonio arquitectónico es usándolo, dándole un rol activo en la sociedad de modo que se valore, se asimile como propio y, como consecuencia, se conserve; no como objetivo a contemplar, sino por el significado social que genera y representa, llegando incluso a propiciar un desarrollo más sostenible.

Dentro de este contexto se encuentra el concepto de reutilización adaptativa, que Douglas (2006) y Yung y Chan (2012) definen genéricamente como "todo trabajo de construcción e intervención para cambiar la capacidad de un inmueble o la función, o las actuaciones para adecuar, reutilizar o actualizar un edificio para adaptarse a nuevas condiciones de uso". De esta manera, se relacionan los conceptos de sostenibilidad con el de conservación de los edificios.

La idea de sostenibilidad en el contexto de la conservación del patrimonio queda definida, al igual que en otros ámbitos, por el conjunto de las componentes social, económico, y ambiental. En el ámbito social se hace hincapié en el papel de 
cohesión de la comunidad (Pendlebury et al., 2004; Yung et al., 2011), en el 'espiritu del lugar' (ICOMOS, 2008) o el refuerzo de la identidad y la conexión con sus raíces (Stubbs, 2004). El componente económico hace referencia a la eficiencia económica, la creación de puestos de trabajo creados por el nuevo uso que contribuyen a las economías locales, la autofinanciación a través de actividades comercialmente viables (Nasser, 2003) o la creación de oferta turística que aporte ingresos a la zona (English Heritage, 1997; Feilden y Jokilehto, 1998). El ámbito ambiental se refiere a los beneficios que repercuten en el medio ambiente al evitar la demolición y posterior construcción en términos de ahorro de energía, la reducción de gases de carbono (Australia Department of Environmnet and Heritage, 2004), el mantenimiento de la trama urbana de la zona y la conservación de su marco histórico (Steinberg, 1996; ICOMOS, 1999). En la literatura reciente, se añade un cuarto componente de la sostenibilidad en el contexto de la conservación del patrimonio referido al ámbito político-institucional (Valentin y Spangenberg, 2000; O'Connor, 2006) que hace referencia a las políticas gubernamentales de apoyo y estrategias a nivel local (Steinberg, 1996; Bullen, 2007; Zhang, 2011) y que promuevan la participación de la comunidad en las esfuerzos de conservación (Yueng, 2006).

Desde el punto de vista de la conservación y protección del patrimonio, la reutilización adaptativa de sitios patrimoniales tiene como objetivo, como se ha visto, garantizar una conservación activa de estos bienes ya que, de forma contraria, corren riesgo de degradación y abandono. Más allá de estas consideraciones, hay que tener presente que con una gestión adecuada pueden incluso considerarse como activos de las comunidades locales y ofrecer beneficios económicos, culturales y sociales. En este sentido, los edificios históricos presentan grandes oportunidades para su reutilización, ya que éstos se localizan en lugares privilegiados que permiten desarrollar y revitalizar el lugar, dan una idea del pasado, actúan como símbolo y señas de identidad de las comunidades locales y pueden servir a las necesidades actuales (Bullen y Love, 2010).

Por otra parte, hay que mencionar también que los sitios patrimoniales presentan dificultades para su reutilización ya que su adaptación a las nuevas normativas de uso público suele ser compleja así como la protección contra incendios, accesibilidad e instalaciones, o su adaptación a las innovaciones tecnológicas y de diseño. Todas estas actuaciones deben garantizar en todo momento la integridad, relevancia, representatividad y singularidad del edificio.

El presente trabajo versa sobre el potencial del Fuerte español de Santiago (s.XVI) en la isla de Chikly (Túnez) para su reutilización como contenedor de actividades recreativointerpretativas.

\section{Metodología}

Para abordar el proceso de reutilización del Fuerte de Santiago se ha aplicado una serie de herramientas secuenciadas en tres fases (fig.1).

En la primera fase, se contemplan todas aquellas acciones de conservación y protección del patrimonio. Todos los trabajos referidos a restauración habían sido ya abordados en años previos por la Agencia Española de Cooperación Internacional para el Desarrollo (AECID) en colaboración con el gobierno tunecino (Instituto Nacional de Patrimonio de Túnez), en un proyecto dirigido por el Dr. José Luis García Grinda, quien documento ampliamente este monumento (García Grinda, 2003).

Así pues, en este proyecto se llevó a cabo la realización de un inventario de los recursos implicados y su posterior valoración teniendo en cuenta su valor intrínseco y su valor educativointerpretativo $\mathrm{y}$ otros valores recreativos. También se realizó la jerarquización y zonificación de los diferentes espacios para disponer de información suficiente para tomar decisiones acerca de su restauración o rehabilitación de su posible utilización para el uso público.

En la segunda fase, se desarrollaron acciones de puesta en valor de los recursos a través del desarrollo de herramientas de accesibilidad 
física (adaptaciones arquitectónicas, desarrollo de equipamientos, estudio de capacidad de carga recreativa, diseño del patrón de la visita, etc.) y se sentaron las bases de las acciones de accesibilidad intelectual y emocional (programa de interpretación).

La tercera fase se dedica a todas aquellas herramientas relacionadas con la dinamización turística, en especial con el diseño de productos turísticos y con la promoción y comercialización de los mismos. Esta fase todavía no ha sido desarrollada en el proyecto, a que exige que las previamente mencionadas estén concluidas.

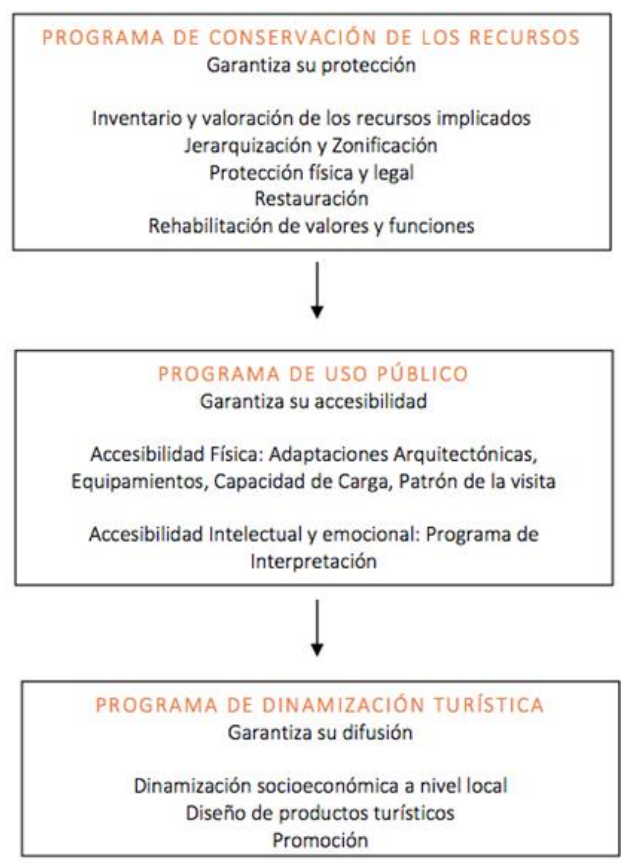

Figura 1. Esquema de las diferentes fases en el proceso de puesta en valor.

\section{El Fuerte de Santiago}

El Fuerte de Santiago se sitúa en la Isla de Chikly (Túnez), localizada en la Laguna Norte (humedal incluido en 2013 en el Listado de Humedales de Importancia Internacional del Convenio de Ramsar). Su ubicación, junto a la ciudad de Túnez, ha hecho que sea un enclave estratégico a lo largo de la historia. Chikly es una pequeña isla de 3 ha declarada Reserva Natural debido a su interés ornitológico. Desde la Antigüedad ha sido un reclamo como hábitat para el hombre, como atestiguan los restos fenicios, púnicos, romanos, bizantinos, árabes, españoles y turcos hallados en ella, siendo el recurso patrimonial más significativo destacado el Fuerte español de Santiago.

El Fuerte, declarado Monumento Histórico en 1992, es una de las obras arquitectónicas más importantes del siglo XVI de Túnez. Fue mandado construir por el emperador Carlos I de España en 1535. Pocos años después, en 1574, la flota turca arrebató a los españoles sus posesiones, siendo el Fuerte de Santiago el último bastión en rendirse. El escritor español Miguel de Cervantes, que participó en la expedición de Don Juan de Austria, narra la caída de Túnez, en su libro "El Quijote", haciendo referencia específica a Chikly.

Tras este suceso, el fuerte sufrió diversas vicisitudes siendo destruido y abandonado definitivamente en el siglo XIX. Ya en el siglo XXI, tal como se ha comentado, fue restaurado, finalizando las obras en 2010. Desde entonces, todavía no tiene asignado un nuevo uso.

El gobierno tunecino, consciente del valor del Fuerte de Santiago y de la Isla de Chikly, así como de su potencial para el desarrollo de actividades culturales y turísticas y, tras varios análisis de la laguna y la isla, decidió poner en valor el Fuerte de Santiago y su entorno isleño (fig. 2).

A finales de 2013, Med-INA (Instituto Mediterráneo para la Naturaleza y Anthropos) junto con la Iniciativa para los Humedales Mediterráneos (MedWet) del Convenio de Ramsar y con la financiación de la Fundación MAVA incluyó este lugar en el proyecto "La cultura y las zonas húmedas del Mediterráneo: Utilización de los valores culturales para la restauración de las zonas húmedas", con la intención de demostrar el potencial de los valores culturales en la restauración y manejo de los humedales mediterráneos.

Este artículo forma parte de los resultados de los estudios de puesta en valor realizados en el marco de este proyecto. 


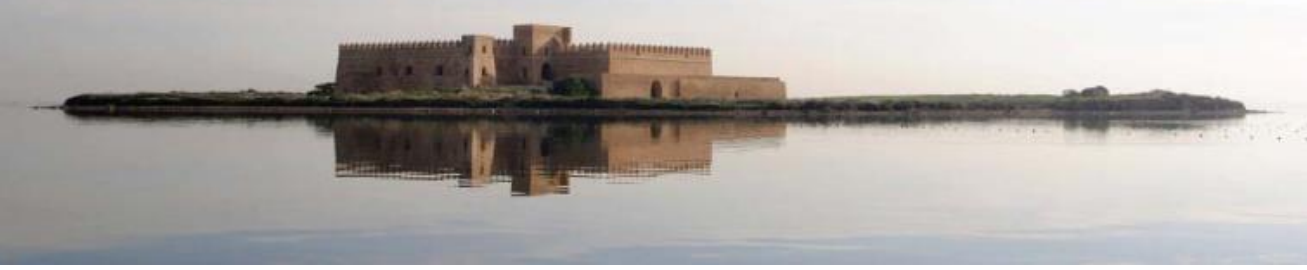

Figura 2. Vista del Fuerte de Santiago desde la embarcación.

\section{Resultados y discusión}

Las herramientas de puesta en valor presentadas en este artículo son las que tienen que ver básicamente con la accesibilidad física al monumento. Previamente, fue necesaria la elaboración del inventario y valoración de los recursos existentes. Los datos obtenidos han permitido conocer en profundidad los elementos que integran el espacio y han facilitado la realización de un diagnóstico del territorio.

En este inventario se incluyó el análisis de la avifauna y la vegetación de la Isla de Chikly, los recursos culturales (restos arqueológicos y Fuerte de Santiago) y también los recursos escénicos (paisajes contenidos en las cuencas visuales) que rodean la laguna y que son observables desde la torre del fuerte.

El sistema de valoración intrínseca y aplicada empleado en este caso de estudio ha sido el mismo que se utilizó en otros casos de estudio con recursos frágiles (Viñals et al., 2013).

La evaluación intrínseca ha determinado el valor de cada recurso a partir de la utilización de criterios como la integridad, significación, representatividad y singularidad.

Por su parte, el proceso de evaluación desde el punto de vista interpretativo determina la aptitud y la idoneidad que los recursos tienen como atractivos para el público o para el desarrollo de ciertas actividades. Un criterio clave utilizado ha sido la fragilidad, ya que informa sobre la respuesta de los recursos frente los impactos derivados de las actividades humanas. También ha sido valorada la atractividad, accesibilidad, disponibilidad en el tiempo y el espacio y la idoneidad de los recursos para la educación e interpretación siguiendo el método empleado en trabajos previos (Viñals et al., 2011).

Del análisis de los valores de los recursos naturales de la Isla de Chikly, se desprende una gran fragilidad ecológica debido a su pequeño tamaño y a la relevancia de sus valores ecológicos, especialmente la avifauna. Hay que destacar asimismo la gran fragilidad visual que presenta debido a su reducido tamaño y a su topografía plana.

La disponibilidad en el tiempo de este espacio es también limitada, ya que existen restricciones estacionales para la visita marcadas por el periodo de reproducción y nidificación (marzojunio) de la garceta común (Egretta garzetta) que presenta en esta isla la colonia más importante de Túnez y de ahí su reconocimiento como espacio protegido. Durante este periodo, las garcetas son altamente vulnerables y cualquier impacto podría causar el abandono momentáneo o definitivo de los nidos y la consecuente pérdida de las crías por depredación de otras aves.

Por su parte, el Fuerte de Santiago destaca, sobre todo, por su legado histórico y simbólico, su relación con la ciudad desde hace cinco siglos y por su construcción en un enclave singular como una pequeña isla. El inmueble se ha mostrado más resistente que la Reserva Natural debido a los materiales con los que está construido. No obstante, tras la conclusión de los trabajos de restauración en 2010, cierto deterioro es evidente.

En relación a las cuencas visuales, hay que destacar que desde la torre de la fortificación se tienen vistas panorámicas completas de todos los 
alrededores que constituyen un recurso escénico de primera magnitud y que han sido convenientemente puestas en valor (MartínezSanchis, 2015).

Respecto a los riesgos y amenazas a los que están sometidos los recursos culturales, se apunta como riesgo principal una falta de uso y gestión (falta de mantenimiento, falta de seguridad, etc.), especialmente de los elementos inmuebles. Estos hechos han dado lugar a un estado de abandono y un proceso de degradación palpable.

Por otra parte, se ha desvelado en este trabajo que tanto la isla como el fuerte y los restos arqueológicos tienen valores educacionales que pueden ser transmitidos a través de mensajes interpretativos dirigidos tanto a la población local como a los potenciales visitantes.

En definitiva, desde el punto de vista de los recursos, se puede decir que la isla de Chikly con su avifauna y paisajes y el fuerte de Santiago junto con los restos arqueológicos tienen un gran potencial para su puesta en valor ya que tienen fuerza interpretativa debido principalmente a su localización en una pequeña isla, con carácter de hito paisajístico y con un elemento monumental como atractivo principal.
Pese a su potencial interpretativo, quedan diferentes aspectos por plantear como la falta de infraestructuras básicas (energía y agua principalmente) y equipamientos, ya que sólo existe un pequeño embarcadero, no hay sanitarios ni señalización informativa, direccional ni interpretativa tal como ya apuntó Darías (2013). Su accesibilidad para personas con movilidad reducida también es muy restringida.

Tras la valoración de los recursos, se realizó una zonificación para determinar el espacio óptimo donde desarrollar las actividades. En la Isla de Chikly, se han identificado y cartografiado dos zonas de exclusión en relación a las áreas de nidificación de las aves y otra referida a la vegetación de interés, ambas acogen a los recursos más frágiles que hay que proteger.

Respecto al Fuerte de Santiago, se ha realizado una zonificación de los diversos espacios en función de la necesaria seguridad de los visitantes (fig.3).

De esta forma, se observa que las estancias de la planta de acceso no pueden transitarse en su mayoría por la dificultad de acceso (cisterna) y mal estado de conservación (almacén) que presentan.
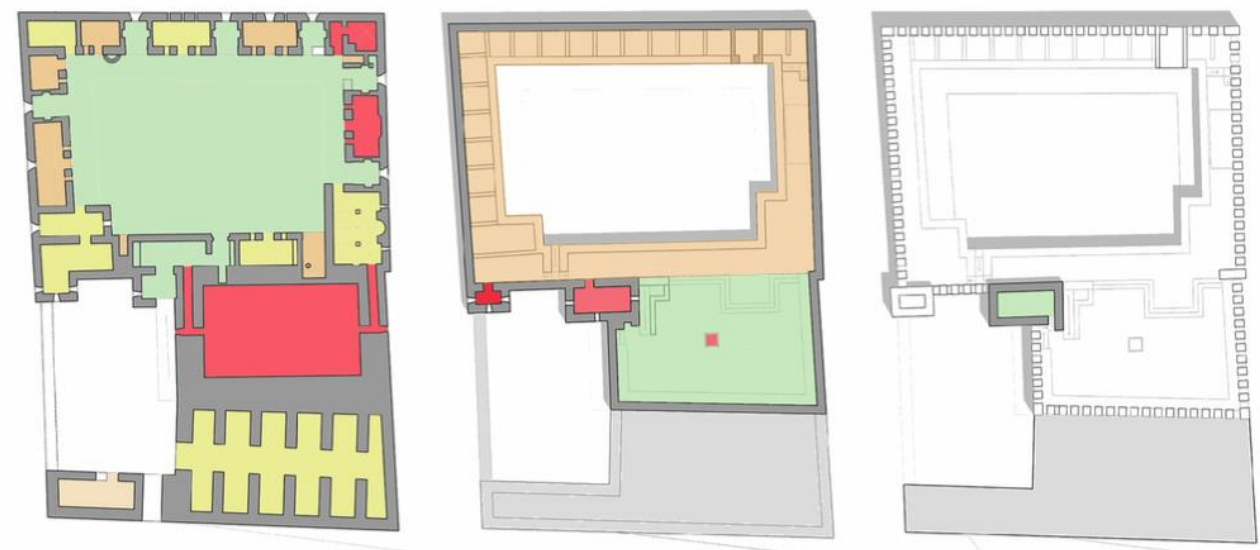

Zona Accesible apta para actividades recreativo-interpretativas Zona con protección. Visibles pero no accesible Zona accesible pero no apta para actividades recreativo-interpretativas

Figura 3. Zonificación del Fuerte de Santiago (Viñals, et al., 2014a) 
En la planta principal, donde se encuentran las estancias en torno al patio, es donde hay más áreas transitables. El patio, con una superficie de $607,2 \mathrm{~m}^{2}$, es totalmente utilizable y también muchas de las salas que lo circundan. No obstante, se ha observado que las aberturas de estas salas tienen que protegerse por razones de seguridad, ya que presentan antepechos muy bajos (entre $20 \mathrm{~cm} \mathrm{y} 60 \mathrm{~cm}$ ) y una anchura grande $(60-65 \mathrm{~cm})$, lo que las hace fácilmente escalables con riesgo de caída al exterior.

Las salas más idóneas para la actividad por razones de acceso, incidencia de la luz solar y zonas de paso y circulación, son los espacios artilleros.

La planta de cubierta presenta una merma de la superficie pisable debido a la falta de barandillas en los remates de los muros del patio principal.

Hay que mencionar también las dificultades de acceso tanto a la planta de cubierta como a la terraza de la torre debido a la existencia de una única escalera utilizable que solo presenta 0,70 $\mathrm{cm}$ de anchura y con peldaños muy altos, lo que solo permite un flujo de usuarios en un único sentido. Por supuesto, queda descartada esta planta para su utilización por personas con movilidad reducida.

Después de realizar la zonificación que ha permitido identificar las áreas idóneas para el uso público $\mathrm{y}$, tras identificar su potencial interpretativo, se diseñó el patrón de la visita. En el momento en el que se realizó el trabajo, las autoridades involucradas no disponían de una identificación clara de las actividades que se querían desarrollar. Hay que comentar que es necesario conocer con detalle las acciones que tendrán lugar durante el desarrollo de la actividad para poder valorar sus necesidades en términos de superficie y tipo de espacio, su incidencia sobre los recursos implicados y el tipo de usuarios que las llevará a cabo.

Fruto del análisis llevado a cabo en este proyecto (Viñals et al., 2014b), diversas actividades recreativas fueron sugeridas a las autoridades responsables. Así, considerados los diferentes espacios existentes, la fragilidad, vulnerabilidad y disponibilidad de los recursos y también considerando la viabilidad administrativa para llevar a cabo estas actividades, se propusieron las siguientes actividades: a) ruta interpretativa en la Reserva Natural de la Isla Chikly; b) Visita interpretativa al Fuerte de Santiago; y c) observación de Aves de la Laguna Norte de Túnez.

La combinación de las tres actividades ofrece un producto interpretativo-educativo completo, pero nunca se podrá ofrecer sola la ruta interpretativa a la Reserva Natural de la Isla de Chikly, pues supondría una carga excesiva para este entorno (Viñals et al., 2014a). Tanto la visita interpretativa al Fuerte de Santiago como la actividad de observación de aves se pueden realizar sin una secuencia establecida y de forma autoguiada. La única actividad que se plantea con secuencia y guiada es la ruta interpretativa en la Reserva Natural de la Isla de Chikly, ya que se trata del recurso más vulnerable y sólo se dispone de los senderos existentes para realizar dicha actividad, cosa que obliga a establecer un solo sentido de circulación en el recorrido y requiere extremar las medidas preventivas para no causar impactos a las aves y la vegetación, por ello debe ser guiada.

Otra herramienta de planificación y gestión turística utilizada en este caso de estudio fue el cálculo de la capacidad de carga recreativa (Viñals et al., 2014a) que una de las herramientas más conocidas que garantizan la protección del sitio patrimonial y la calidad de la experiencia del visitante.

Los resultados apuntaron a que la isla es mucho más frágil que el Fuerte y que, por tanto, limita el número de personas que pueden visitar el conjunto. Según los cálculos obtenidos, pueden visitar la Isla de Chikly y el Fuerte de Santiago 20 personas a la vez pero divididas en dos grupos independientes, siendo los principales factores limitantes los ecológicos, tal como se ha comentado, debido a la nidificación de las aves en la superficie de la isla y a la fragilidad visual que ésta presenta al ser una superficie plana. 


\section{Conclusiones}

El análisis del Fuerte de Santiago, tras su restauración en 2010, nos informa que la no utilización del edificio ha conllevado un deterioro evidente.

Otra reflexión oportuna es que no se puede abrir al público para su visita si previamente no se garantizan las medidas de conservación y protección necesarias que garanticen la integridad del edificio.

También es necesario destacar que la reutilización adaptativa como es el caso del Fuerte, precisa la realización de una serie de adaptaciones físicas para asegurar la seguridad y confort de los visitantes. También debe acometerse el desarrollo de un adecuado programa de interpretación que dote a este sitio patrimonial del acceso intelectual que un monumento de su categoría merece.

La reutilización para la visita pública del Fuerte de Santiago no constituirá un problema de conservación de este edificio histórico sino que contribuirá a garantizar su mantenimiento y así evitar una degradación irremediable si no se ponen en marcha otras medidas de conservación activas. De cualquier manera hay que señalar que la Isla de Chikly se ha mostrado extremadamente frágil y esto condiciona, en gran manera, la visita al Fuerte, por lo que la gestión de ambos bienes patrimoniales se ha de llevar a cabo de forma conjunta y coordinada

\section{Referencias}

Australia Department of Environment and Heritage (2004). Adaptive Reuse. Commonwealth of Australia, Canberra.

Bullen, P.A. (2007). Adaptive reuse and sustainability of commercial buildings. Facilities, 25, pp.20-31.

Bullen, P.A. y Love, P.E.D. (2010). The rhetoric of adaptive reuse or reality of demolition: views from the field. Cities, 27, pp.215-224.

Canada's Historic Places (2010). Standards and Guidelines for the Conservation of Historic Places in Canada. Technical report, $288 \mathrm{pp}$.

Chapple, N. (2012). New uses for former places of Worship. Technical report. English Heritage, 20 pp.

Darías, A. (2013). "El castillo de la Isla de Chikly: una propuesta para el turismo cultural sostenible", in NORBA Revista de Arte, vol. XXXII-XXXIII, pp. 285-300

Douglas, J. (2006). Building adaptation (2nd ed.). Butterworth-Heinemann, 651pp.

English Heritage. (1997). Sustaining the historic environment. London: English Heritage, 17 pp.

Feilden, B. M., y Jokilehto, J. (1998). "Evaluation for conservation" in Management guidelines for world heritage sites, pp.11-21.

García Grinda, J.L. (2003). Santiago de Chikli: una fortaleza española en Túnez. Ed. MAE-AECI, 64 pp.

ICOMOS (1999): The Burra charter, the Australia ICOMOS charter for places of cultural significance.

ICOMOS (2008): Québec Declaration on the preservation of the Spirit of place.

Martínez-Sanchis, I. (2015), Propuesta para la puesta en valor del Fuerte español de Santiago (s.XVI) en la Isla de Chikly, Túnez. Tesina de Master. Universitat Politècnica de València (Inédito).

Nasser, N. (2003). "Planning for urban heritage places: reconciling conservation, tourism, and sustainable development", in Journal of Planning Literature, 17, pp.467-479.

O'Connor, M. (2006). "The "Four Spheres" framework for sustainability" in Ecological Complexity, 3 , pp.285-292.

Pendlebury, J., Townshend, T. y Gilroy, R. (2004). "The conservation of cultural built heritage: a force for social inclusión", in International Journal of Heritage Studies, 10, pp.11-31.

Steinberg, F. (1996). "Conservation and rehabilitation of urban heritage in developing countries" in .Habitat International, 20, pp.463-475.

Stubbs, M. (2004). Heritage-sustainability: developing a methodology for the sustainable appraisal of the historic environment. Planning Practice \& Research, 19, pp.285-305. 
UNESCO (1964). Cartas y Recomendaciones Internacionales para la Conservación y Restauración del Patrimonio Cultural. 'La carta de Venecia.

UNESCO (1972). Convención sobre la protección del patrimonio mundial, cultural y natural. Conferencia General de la Organización de las Naciones Unidas para la Educación, la Ciencia y la Cultura, 17a, reunión.

Valentin, A. y Spangenberg, J. H. (2000). A guide to community sustainability indicators. Environmental Impact Assessment Review, 20, pp.381-392.

Viñals, M.J., Morant, M. y Quintana, R. (2011). Análisis de los criterios para la valoración turística del patrimonio natral. Investigaciones turísticas, 1, pp.37-50.

Viñals, M.J., Alonso-Monasterio, P. y Alonso-Monasterio, M. (2013). Analysis of the Spatial Standards and Perceptual Components of the Recreational Carrying Capacity Applied to Archaeological Sites: Case Study of Castellet de Bernabé (Llíria, Spain). En: Mondéjar, Vargas Ortega y Perez Calderon (eds.): Methods and Analysis on Tourism and Environment. Ed. Nova Science Publishers (New York), pp.109-120.

Viñals M.J., Martínez-Sanchis, I., Abdennadher, A. y Teruel, L., (2014a). A recreational carrying capacity assessment of the 16th century Fort of Santiago on the Island of Chikly, Tunisia. WIT Transaction on The Built Environment, Vol 143, pp.185-196.

Viñals, M.J., Martínez-Sanchis, I., Abdennadher, A., Morant, M. y Teruel, L. (2014b). Étude d'évaluation de la capacité d'accueil de l'île chikly et de ses environs. Universitat Politècnica de València (Inédito).

Yuen, B. (2006). Reclaiming cultural heritage in Singapore. Urban Affairs Review, 44, pp. 830-854.

Yung, E.H.K. y Chan, E.H. (2012). Implementation challenges to the adaptive reuse of heritage buildings: Towards the goals of sustainable, low carbon cities, Journal of Habitat International, 36, pp. 352-361.

Zhang, Y. (2011). Boundaries of power: politics of urban preservation in two Chicago neighborhoods. Urban Affairs Review, 47, pp. 511-540. 